Christian Mester*

\title{
Optimised calibration programmes for comparators for instrument transformers
}

\author{
Technologieoptimiertes Kalibrierprogramm für Wandlermessgeräte
}

\author{
Using new technologies to the full extent thanks to traceable calibration
}

\author{
Neue Möglichkeiten durch Kalibrierung tatsächlich nutzbar machen
}

https://doi.org/10.1515/teme-2020-0086

Received November 14, 2020; accepted December 21, 2020

\begin{abstract}
Traditionally, instrument transformers are calibrated using bridges. By definition, bridges use the null method of measurement. The traditional calibration programme for instrument transformer bridges characterise namely this null measurement. Many new commercial comparators for instrument transformer use a very different method. They sample the secondary signals of reference and device under test (DUT) transformer independently. Based on the samples, magnitude and phase of both signals are determined. Ratio error and phase displacement are calculated. Consequently, the significance of their calibration using the traditional calibration programme is limited. Moreover, the operating range of modern comparators is much larger than that of bridges. The additional versatility cannot be used without an adapted calibration programme. This article analyses the calibration programmes for both technologies. An experimental study confirms both the suitability of the new calibration programme and the need to chose the calibration programme depending on the technology of the device to be calibrated. The conclusion is very general and applies to all measurement problems where an operating principle is replaced by another - when changing the operating principle, it is important to check the calibration programme and adapt it if necessary.
\end{abstract}

Keywords: Calibration, bridge, comparator, measurement uncertainty, instrument transformer, instrument transformer test set.

Zusammenfassung: Traditionell werden bei der Kalibrierung von Messwandlern Messbrücken verwendet. Brücken verwenden definitionsgemäß ein Verfahren mit Nullab-

\footnotetext{
*Corresponding author: Christian Mester, Federal Institute of Metrology METAS, Lindenweg 50, 3003 Bern, Switzerland, e-mail: christian.mester@metas.ch, ORCID: https://orcid.org/0000-0002-2773-3883
}

gleich. Durch das klassische Kalibrierprogramm wird insbesondere dieser Nullabgleich charakterisiert. Moderne Wandlermessgeräte hingegen tasten die Ausgangssignale von Referenz und Prüfling ab, bestimmen aus den Abtastwerten Effektivwert und Phase der beiden Signale und berechnen Messabweichung und Fehlwinkel hieraus. Folglich ist eine Kalibrierung mit dem klassischen Kalibrierprogramm für Wandlermessbrücken für solche Geräte nur bedingt aussagekräftig. Zudem bieten solche Geräte eine wesentlich größere Flexibilität im Einsatz, die durch klassische Kalibrierprogramme jedoch nicht abgebildet wird. Hier werden verschiedene Kalibrierprogramme für diese beiden Technologien analysiert. Eine experimentelle Studie bestätigt einerseits deren Eignung und andererseits die Notwendigkeit, das Kalibrierprogramm technologieabhängig zu wählen. Die Schlussfolgerung ist sehr grundsätzlich und auf alle Messaufgaben anwendbar, bei denen ein Funktionsprinzip durch ein anderes ersetzt wird - wird das Funktionsprinzip geändert, so muss geprüft werden, ob auch das Kalibrierprogramm angepasst werden muss.

Schlagwörter: Kalibrierung, Messunsicherheit, Messwandler, Komparator, Messbrücke, Wandlermessgerät, Wandlermessbrücke.

\section{Introduction}

Traditionally, instrument transformers are calibrated using bridges. By definition, bridges use the null method of measurement (Fig. 1a) [1]. Some practical realisations use a differential method of measurement [1] instead, where the complex difference between the reference and the DUT signal is measured directly, without adjustment to zero. Please note that comparators have at least two channels, usually labelled "reference" and "DUT". Therefore, this paper uses the abbreviation DUT only in relation to the comparator's channel labelled DUT, even though the device 
whose calibration is discussed here is the comparator, not the transformer connected to the comparator's DUT channel. The traditional calibration programme for instrument transformer bridges characterises the difference measurement and the normalisation to reference amplitude. During the calibration, the complex error is varied while keeping the reference signal at a constant amplitude. In a second step, the excitation, i. e. the amplitude of the signal normalised to its rated value, is varied while the error is zero. The latter part is very easy to realise, since it is sufficient to apply the same signal to both the reference and the DUT channel. To vary the complex error, an error generator is required. The error generator emulates an instrument transformer with adjustable complex error in the DUT channel [2]. The complex error is usually specified and displayed in terms of ratio error and phase displacement, as defined in IEC 61869-1 [3]. One very convenient property of this is that the settings of the error and the display of the bridge are in terms of the same quantities, excitation, ratio error and phase displacement. A significant disadvantage is that bridges cannot be used when the transformation ratio of reference transformer and DUT transformer differ. Commercial bridges avoid this problem with adaptation transformers within the bridge, adding some flexibility at the expense of a more complicated calibration. However, when the DUT transformer is a low-power instrument transformer or has a digital output - for instance according to IEC 61850-9-2 [4] - bridges are inconvenient, if not impossible to use. When calibrating high-accuracy reference transformers in national metrology institutes such as the Federal Institute of Metrology METAS, bridges are still the preferred choice. They can be designed for such transformers with differences below $50 \times 10^{-6}$ and reach uncertainties below $0.01 \times 10^{-6}$. Most participants in the last European current transformer comparison used bridges [5].

Nowadays, most new measuring instruments for the calibration of instrument transformers - even though they are often sold as bridges - are not bridges anymore. To reflect this, the IEC introduced the general term comparator [6]. Those comparators using an indirect method of measurement [1] sample both analogue input channels (Fig. 1b). The sampling of the channels is independent, only the triggering is synchronised. It is easily possible to have very different input signals. When calibrating a current transformer, the reference transformer's secondary signal is usually a current. The DUT could be a low-power instrument transformer LPIT with a secondary voltage. As long as the synchronisation is maintained, it is possible to move the analogue-to-digital converter into the DUT, making the interface digital. Moreover, since there is no difference being directly measured, arbitrary phase displace- ments are permissible without any impact on the uncertainty. A practical case is the calibration of a Rogowski coil [7] as DUT, where the rated phase displacement is $90^{\circ}$. Another example is the calibration of an inverting DUT, where the amplitude of the difference signal would be as large as the sum of the amplitudes of the secondary signals of reference and DUT, exceeding the operating range of a welldesigned bridge by far.

In principle, comparators using an indirect method of measurement can also be used to calibrate instrument transformers for power quality measurements [8]. While commercial comparators do not include this feature yet, METAS set up and calibrated such a comparator along with the appropriate sources.

It is possible and unfortunately very common to maintain the traditional calibration programme when replacing bridges by comparators using an indirect method of measurement without giving it much thought. The lack of a term as concise and established as bridge incites the use of the wrong term bridge by commercial vendors. In this confusion, it is commonly overlooked that the usable operating range becomes strongly limited by the calibration programme.

This article analyses the operating principles of traditional bridges and comparators using an indirect method of measurement theoretically. This article shows that it is possible to calibrate the complete operating range of such a comparator without increasing the number of calibration points. It also shows how the usable operating range would be limited by the traditional calibration programme for bridges. Furthermore, it proposes to replace the traditional, extensive calibration by a short calibration and an extensive type test. The calibration characterises properties, mainly in the analogue domain, that are subject to ageing, wear and tear. The type test characterises properties, mainly due to software, that are equally important, but do not change with time. While the calibration needs to be repeated regularly, the type test does not. An experimental study confirms the assumptions.

\section{Traditional comparators: bridges}

Signals in comparators for instrument transformers can be represented as vectors (Fig. 2). The input signal in the reference channel, i. e. the secondary signal of the reference transformer, is labelled $N$, that in the DuT channel $X$. The difference of the angle of $X$ with respect to $N$ is called phase displacement $\Delta \varphi$, where $\Delta \varphi$ is defined to be positive when $X$ is leading with respect to $N$. In bridges, the 


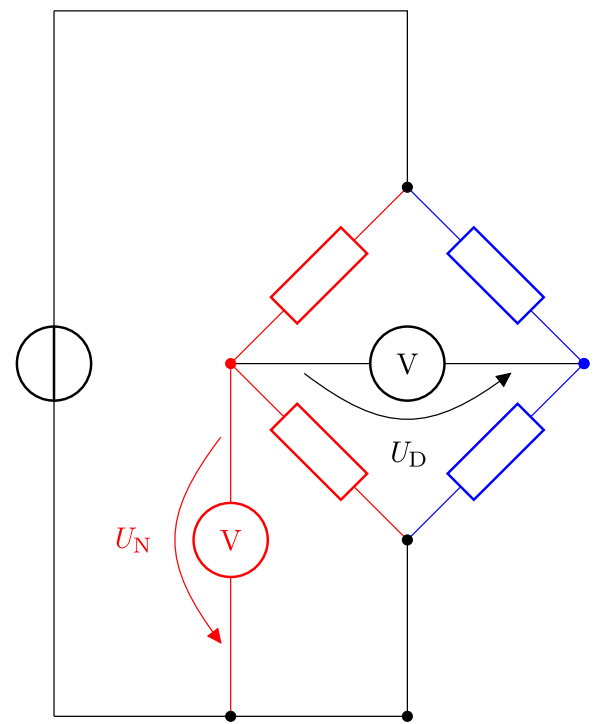

(a) Bridge: null method of measurement (adjustment not shown)

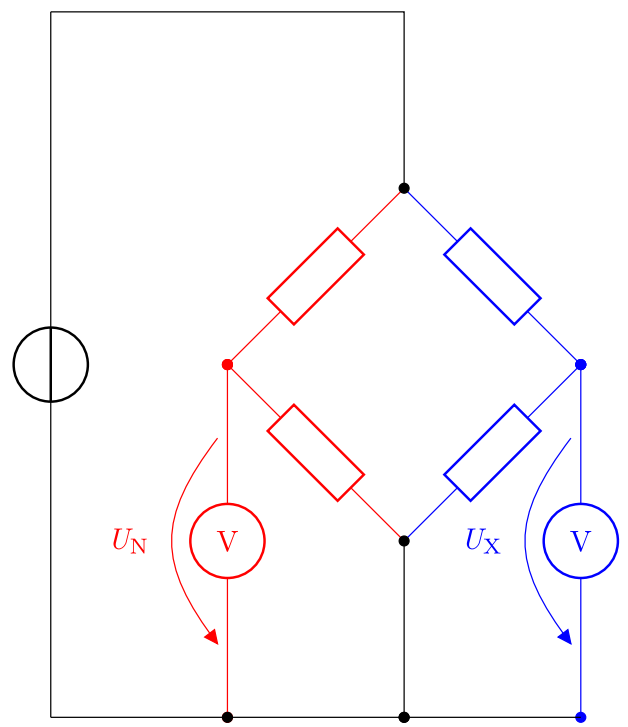

(b) Indirect method of measurement

Figure 1: Operating principles. For simplicity, the instrument transformers are replaced by pairs of resistors here.

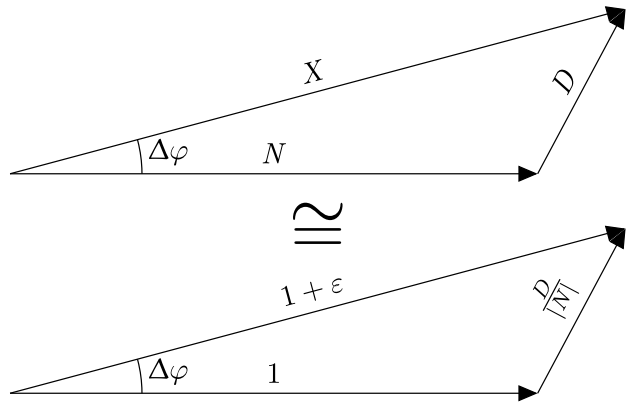

Figure 2: Vector diagram: Signals in bridges.

difference $D:=X-N$ is realised in hardware. The three vectors $N, X$ and $D$ define a triangle. Normalisation yields a congruent triangle from which the ratio error $\varepsilon$ can be determined easily. For the following analysis, the difference between comparators using a null method of measurement (bridges) and comparators using a differential method of measurement is irrelevant. Therefore, these two methods are treated as one for simplicity. The vector diagram shows clearly that bridges can only be used if $\varepsilon$ and $\Delta \varphi$ are suffiently small to avoid overdriving the element measuring $D$. In the extreme case of $X=-N,|D|=2|N|$. Commercial bridges are usually protected against damage resulting from this case. However, they are not designed to measure under such conditions because if they were, their resolution would be insufficient for the small values of $\varepsilon$ and $\Delta \varphi$ they are intended to measure. Usually, commercial bridges include adaptation transformers to allow a prede- fined mismatch between $N$ and $X$, for instance $1: 1 / \sqrt{3}$ for voltage transformers or $5: 1$ for current transformers. Table 1 shows typical specifications of a commercial bridge.

A bridge for current transformers (Fig. 3), for instance, consists of two measuring elements, one for the complex current $I_{\mathrm{N}}$ in the reference channel and one for the complex current $I_{\mathrm{D}}$ in the difference branch. Furthermore, a calculation stage determines the ratio error $\varepsilon$ and the phase displacement $\Delta \varphi$. Since the difference signal $D$ depends on both $\varepsilon$ and $\Delta \varphi$, the measured values of $\varepsilon$ and $\Delta \varphi$ are not independent of each other. Old bridges often use approximations. The calibration needs to cover both measuring elements as well as the calculation stage.

Usually, the whole operating range is calibrated on the two axes with $\varepsilon=0$ and with $\Delta \varphi=0$ (Fig. 4). As a check, it is common to calibrate a very limited number of points with $\varepsilon \neq 0$ and $\Delta \varphi \neq 0$. If the device contains adaptation transformers, these need to be calibrated, too, but since they are not influenced by the other channels' signal, there is no need to vary $\varepsilon$ and $\Delta \varphi$ in this part of the calibration. Furthermore, the zero $(\varepsilon=\Delta \varphi=0)$ is calibrated for all permissible excitations. The latter calibration is very simple, since it is sufficient to apply the same signal to both channels at the same time. This part can be easily repeated by the user as a quick - albeit limited - check showing that the bridge's characteristics influencing this measurement did not change since the last calibration. However, this check does not replace regular calibrations - the linear- 
Table 1: Typical specifications of a commercial bridge.

\begin{tabular}{ll}
\hline Input voltage & $3 \mathrm{~V}$ to $400 \mathrm{~V}$ \\
Input impedance & $<1 \mathrm{VA}$ at $100 \mathrm{~V}$, corresponding to $>10 \mathrm{k} \Omega$ \\
Ratio error $\varepsilon$ : Ranges & $-19.99 \%$ to $19.99 \%$ \\
& $-1.999 \%$ to $1.999 \%$ \\
& $-0.1999 \%$ to $0.1999 \%$ \\
Phase displacement $\Delta \varphi$ : Ranges & $-19.99 \mathrm{crad}$ to $19.99 \mathrm{crad}\left(-687^{\prime}\right.$ to $\left.687^{\prime}\right)$ \\
& $-1.999 \mathrm{crad}$ to $1.999 \mathrm{crad}\left(-68.7^{\prime}\right.$ to $\left.68.7^{\prime}\right)$ \\
& $-0.1999 \mathrm{crad}$ to $0.1999 \mathrm{crad}\left(-6.87^{\prime}\right.$ to $\left.6.87^{\prime}\right)$ \\
\hline
\end{tabular}

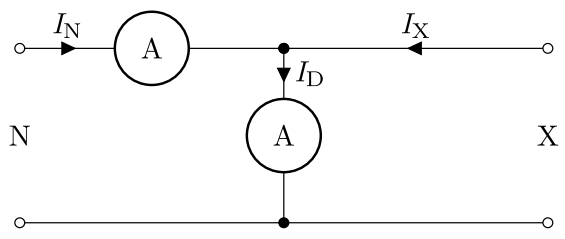

Figure 3: Bridge for current transformer calibration.

ity of the difference measurement, for instance, is essential for the performance of the bridge, but is not checked in this way.

\section{Comparators using an indirect method of measurement}

Comparators using an indirect method of measurement can be used in the same way as bridges, but they are much more versatile. As shown in the block diagram (Fig. 5), they determine amplitude and phase of the reference $(N)$ and the DUT $(X)$ signal independently. They do not realise the difference signal in hardware. Therefore, there is no limitation on the difference signal. The worst case for a traditional bridge, $X=-N$, i. e. $|D|=2|N|$, can be measured with the same uncertainty as the best case $X=N$, i. e. $D=0$. Thanks to modern electronics, they reach uncertainties that are comparable to those of commercial bridges and exceed the requirements of commercial applications (Tab. 2).

It is possible to use the calibration programme for bridges, which has been used for decades and is generally accepted, since the signal levels used during calibration are covered by the operating range. However, the operating range is much larger. This calibration programme would only cover a very small part of it, thereby limiting the usable operating range significantly. The increased versatility of the comparator could not be used. In addi-
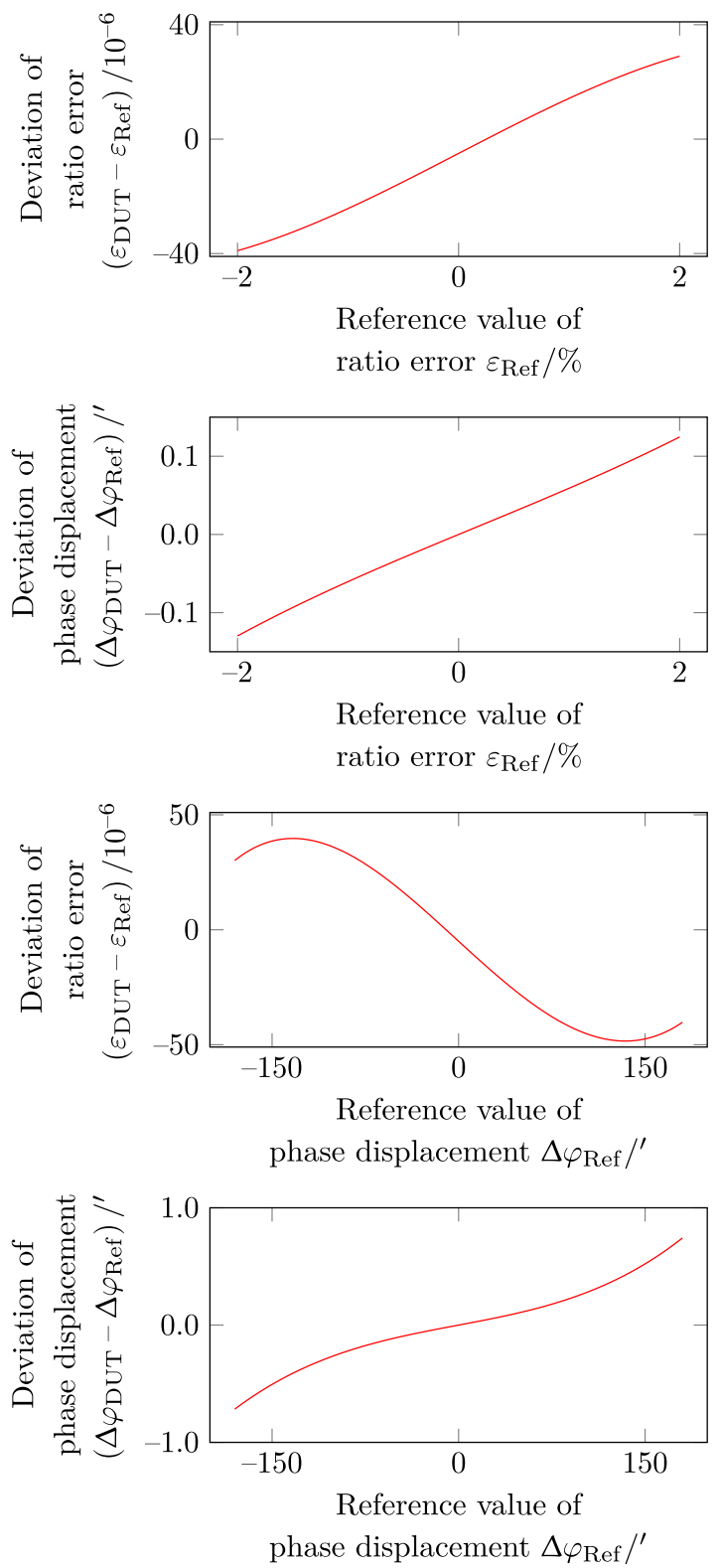

Figure 4: Calibration of a bridge: simulation results. Typical uncertainties $(k=2)$ in real calibrations: $26 \times 10^{-6}$ and $0.09^{\prime}$. 
tion, the calibration programme would contain many redundant points.

\subsection{Calibration of the ratio error $\varepsilon$}

Calibration programmes for bridges contain a large number of points where the ratio error $\varepsilon$ is varied in small steps for a constant reference signal $N$. The specification requires $|\varepsilon|$ to be very small, e. g. $|\varepsilon|<2 \%$, hence $X \approx N$. For comparators using an indirect method of measurement, this means that the linearity of the $X$ channel is analysed in great detail for small-signal excitation around a fixed operating point. If the analogue front-end including the analogue-to-digital converter is designed properly, the deviation of the comparator is constant for all $\varepsilon$ of this calibration programme (Fig. 6). In other words, a single calibration point would have given the same information

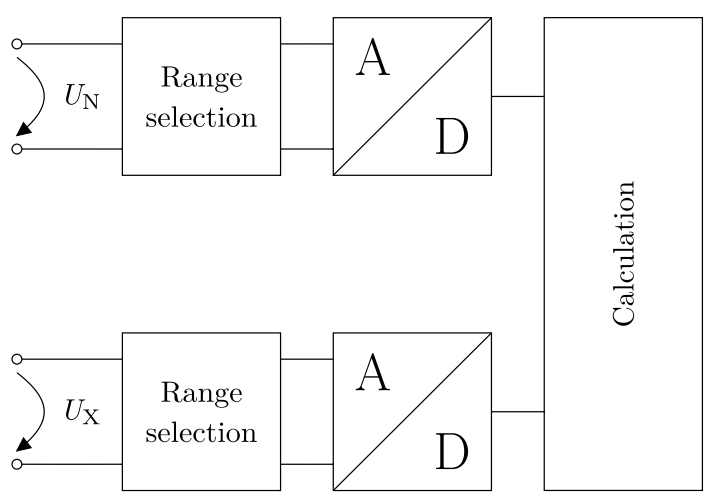

Figure 5: Comparator for voltage transformers using an indirect method of measurement. without loss of quality. A useful addition to the calibration programme would be a characterisation of the linearity of the front end across a much larger range, ideally covering the whole operating range. This point is very relevant when reference and DUT transformers have different transformation ratios. Especially dangerous is the false conclusion that given the result of the limited calibration for $X \approx N$ and the absence of adaptation transformers, the behaviour of the comparator was the same across the whole operating range. This argumentation overlooks that these comparators usually use multiple different measuring ranges. Within the ranges, the linearity is usually excellent, but between ranges, steps occur (Fig. 7). Therefore, the calibration programme must be extended to include all ranges. Since the comparator calculates the ratio error $\varepsilon$ based on the measured amplitudes of $N$ and $X$, it is meaningful to specify the applied amplitudes $N$ and $X$ in the calibration certificate rather than the - very large values of $\varepsilon$.

\subsection{Calibration of the phase displacement $\Delta \varphi$}

Bridges can only be used for small phase displacements $\Delta \varphi$. Consequently, the rated phase offset of the reference and the DUT transformer must be equal. Usually, this is a trivial requirement - bridges are usually designed and used for inductive instrument transformers only. Their rated phase offset is zero by definition [3]. Other instrument transformers than inductive transformers can have any rated phase displacement; for Rogowski coils [7], it is $90^{\circ}$. Since such transformers - the discussion about

Table 2: Typical specifications of a commercial comparator using an indirect method of measurement.

\begin{tabular}{|c|c|c|}
\hline Reference channel & $\begin{array}{l}\text { Ranges } \\
\text { Input impedance }\end{array}$ & $\begin{array}{l}500 \mathrm{~V}, 250 \mathrm{~V}, 125 \mathrm{~V}, 60 \mathrm{~V}, 30 \mathrm{~V}, 15 \mathrm{~V}, 7.5 \mathrm{~V} \text { and } 3.75 \mathrm{~V} \\
380 \mathrm{k} \Omega, 500 \mathrm{pF}\end{array}$ \\
\hline DUT channel 1 & $\begin{array}{l}\text { Ranges } \\
\text { Input impedance }\end{array}$ & $\begin{array}{l}500 \mathrm{~V}, 250 \mathrm{~V}, 125 \mathrm{~V}, 60 \mathrm{~V}, 30 \mathrm{~V}, 15 \mathrm{~V}, 7.5 \mathrm{~V} \text { and } 3.75 \mathrm{~V} \\
380 \mathrm{k} \Omega, 500 \mathrm{pF}\end{array}$ \\
\hline DUT channel 2 & $\begin{array}{l}\text { Ranges } \\
\text { Input impedance }\end{array}$ & $\begin{array}{l}15 \mathrm{~V}, 10 \mathrm{~V}, 5 \mathrm{~V}, 2.5 \mathrm{~V} \text { and } 1 \mathrm{~V} \\
500 \mathrm{mV}, 250 \mathrm{mV}, 100 \mathrm{mV}, 50 \mathrm{mV} \text { and } 25 \mathrm{mV} \\
>1 \mathrm{G} \Omega, 70 \mathrm{pF}\end{array}$ \\
\hline Digital channel & & according IEC 61850-9-2 \\
\hline Tolerance on $\varepsilon$ and $\Delta \varphi$ & $\begin{array}{l}\text { DUT channel } 2 \\
\text { Digital DUT }\end{array}$ & $\begin{array}{ll}200 \times 10^{-6}, 200 \mu \mathrm{rad}\left(0.69^{\prime}\right) & \text { at } 2<U_{\mathrm{N}} / U_{\mathrm{X}} \\
100 \times 10^{-6}, 100 \mu \mathrm{rad}\left(0.34^{\prime}\right) & \text { at } 1.1<U_{\mathrm{N}} / U_{\mathrm{X}} \leq 2 \\
50 \times 10^{-6}, 50 \mu \mathrm{rad}\left(0.17^{\prime}\right) & \text { at } 0.9 \leq U_{\mathrm{N}} / U_{\mathrm{X}} \leq 1.1 \\
100 \times 10^{-6}, 100 \mu \mathrm{rad}\left(0.34^{\prime}\right) & \text { at } 0.5 \leq U_{\mathrm{N}} / U_{\mathrm{X}}<0.9 \\
200 \times 10^{-6}, 200 \mu \mathrm{rad}\left(0.69^{\prime}\right) & \text { at } U_{\mathrm{N}} / U_{\mathrm{X}}<0.5 \\
400 \times 10^{-6}, 200 \mu \mathrm{rad}\left(0.69^{\prime}\right) & \\
100 \times 10^{-6}, 300 \mu \mathrm{rad}\left(1.1^{\prime}\right) & \end{array}$ \\
\hline
\end{tabular}



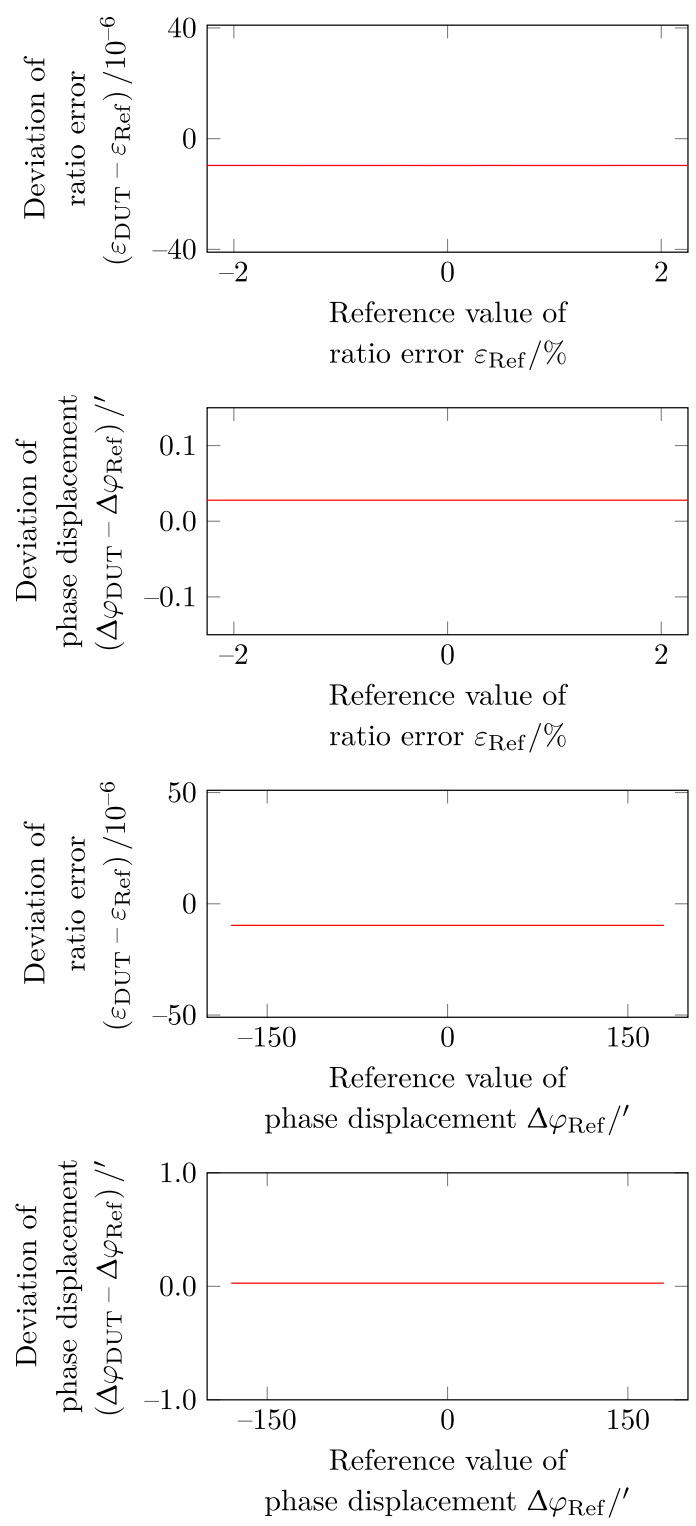

Figure 6: Calibration of a comparator using an indirect method of measurement: simulation results. Typical uncertainties $(k=2)$ in real calibrations: $26 \times 10^{-6}$ and $0.09^{\prime}$.

whether or not those should still be called instrument transformers in the future is still ongoing in IEC TC 38, but current standards use this term - are becoming more and more common, IEC 61869-6 introduced the terminology required to address this. The symbol $\Delta \varphi$ is used for the phase displacement and $\varphi_{\mathrm{e}}$ is the phase error [9]. The most recent draft of IEC 61869-1 [10] uses this convention for all kinds of instrument transformers. Comparators using an indirect method of measurement can be used equally well for any phase displacement $\Delta \varphi$. The phase error $\varphi_{\mathrm{e}}$ is calculated based on the measured phases of $N$ and $X$. This calculation
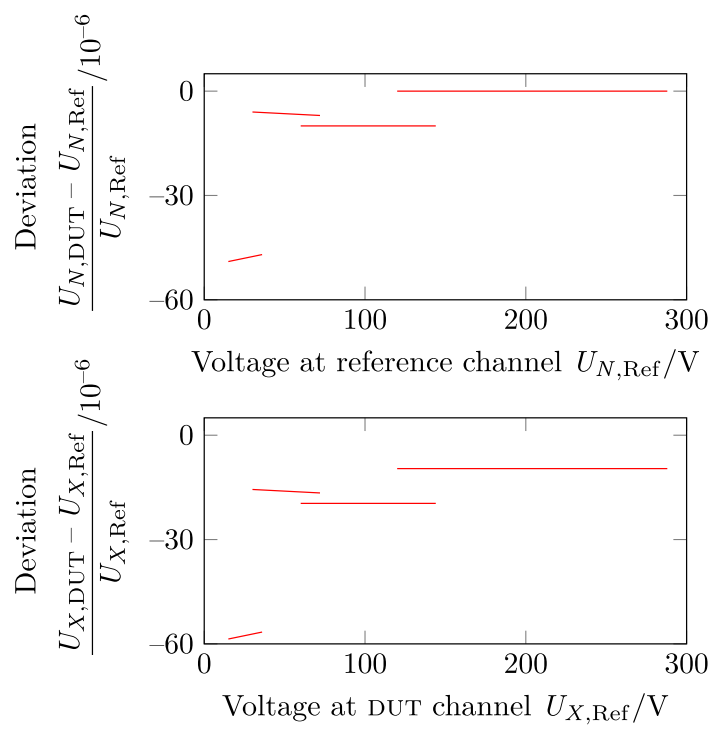

Figure 7: Calibration of a comparator using an indirect method of measurement: simulation results. Typical uncertainties $(k=2)$ in real calibrations: $26 \times 10^{-6}$. Mismatch between channels is negligible, but strong dependency on ranges needs to be accounted for.

is done in software. There is no need to check the correctness of this calculation during every calibration.

For the correct determination of the RMS values, it is not necessary that both signals have the same frequency. In practice, the frequency of the secondary signal of the reference and the DUT transformer is identical by design of the calibration set-up since the primary signal of both transformers is identical. However, differing internal clock frequencies in the measurement elements of the comparator or an unfortunate choice of internal algorithms [11] can cause false frequency differences. This impacts the measurement of the phase displacement $\Delta \varphi$, but not the ratio error $\varepsilon$. The larger the phase displacement, the larger the impact of this effect. Therefore, a characterisation with large phase displacements is necessary once. For small phase displacements, the effect is much smaller than the measurement uncertainty and hence invisible.

Other effects impacting the measurement phase displacement $\Delta \varphi$ are the phase shift in the analogue frontend of the individual channels and delay mismatch in the triggering paths of the analogue-to-digital converters. The phase shift in the analogue front-end can be made constant by careful choice of the components within every range, i. e. independent of the signal amplitude as long as the range is not changed. The delay mismatch in the triggering paths is constant as long as the same analogue-todigital converters are used. Both effects are independent of the ratio error $\varepsilon$ and the phase displacement $\Delta \varphi$. There 
is no need for fine steps of $\varepsilon$ and $\Delta \varphi$ in the calibration of comparators using an indirect method of measurement.

The measurement of the phase displacement $\Delta \varphi$ depends on the linearity of the measuring elements. The phase of a signal can, for instance, be determined from the timing of the first sample after the zero crossing. However, a very high sampling rate is necessary to satisfy the uncertainty requirements. A more appropriate solution is to decompose the sinusoidal signal using the equation $\sqrt{2} N \sin \left(2 \pi f_{0} t+\varphi\right)=N_{1} \sin \left(2 \pi f_{0} t\right)+N_{2} \cos \left(2 \pi f_{0} t\right)$ and to determine the phase from the ratio $N_{2} / N_{1}$. The impact of the linearity of the comparator on the phase difference $\Delta \varphi$ is easily visible when reading $\Delta \varphi$ during the linearity analysis of the ratio error $\varepsilon$. Additional calibration points are not necessary.

For comparators using an indirect method of measurement with analogue inputs only, it is sufficient to know the phase displacement between the two analogue input signals. If only one signal, usually the secondary signal of the reference transformer, is analogue and the other one digital $[12,13]$, the phase measurement of the comparator must use the same timing reference as the DUT transformer. Usually, this is a pulse-per-second (PPS) signal derived from a satellite navigation system. Since both the analogue signal and the external synchronisation signal are delayed in the comparator, separating the contributions of the analogue and the digital path is not trivial $[14,15,16,17,18]$.

\section{Type testing}

A calibration is necessarily limited to a finite number of calibration points, but the operating range of a comparator - and, in general, any real measuring instrument contains an infinite number of possible operating points. Knowledge about the operating principle of the device to be calibrated allows the expert to define a practical calibration programme. Imagine a device to be calibrated that is expected to be linear over its operating range. A calibration with two calibration points at the extrema is sufficient. An additional point between the extrema allows for a simple check of the assumption of linearity. So an expert can calibrate this device with two points; an additional point gives confidence in their claim that two points are sufficient. In principle, the assumption of linearity needs to be checked only once, but since the effort required for a single extra calibration point is very small, it is usually repeated at every calibration. A non-expert, however, would need a calibration programme with a very large number of points. The expert's advantage is even more pronounced when the device to be calibrated has multiple input quantities - like a comparator for instrument transformers. Since the device is subject to ageing as well as wear and tear, the calibration needs to be repeated at intervals.

This article postulates some properties of comparators depending on their internal implementation. This is of some interest to prospective users choosing a comparator to buy. A practical user of a given comparator is less interested in generalities about possible implementations of comparators in general. They need to know as much as possible about their comparator and convince auditors of this knowledge and its correctness. The operating principle does not age and does not wear out. The knowledge about it is valid for the lifetime of the comparator. The intended properties are usually described in the operating manual with sufficient detail, but unintended properties are not. So the user needs a way to determine the relevant properties by observation rather than by declaration of the manufacturer. An efficient solution is the combination of calibrations at regular intervals and a single type test carried out once. The knowledge acquired or confirmed during the type test allows for a reduction of the calibration programme without loss of information. This procedure is well-established in legal metrology, where type approvals are issued based on extensive type tests [19] of a small number of devices - which must be representative for its type - and every instrument is verified with a very small number of verification points [20].

Table 3 gives an overview of properties of comparators using an indirect method of measurement.

\section{Experimental results}

The theoretical analysis discussed above was confirmed experimentally using comparators from different manufacturers. Table 4 gives a summary. Since the extreme values of the deviations are to be found at the extrema of $\varepsilon$ and $\Delta \varphi$, the extreme values are given (rows "max" and "min") together with the values at $\varepsilon=\Delta \varphi=0$ (rows "0"). Bridges are easily identified by the influence of $\Delta \varphi$ on $\varepsilon$ as seen in the column $\varepsilon(\Delta \varphi)$ and $\varepsilon$ on $\Delta \varphi$ as seen in the column $\Delta \varphi(\varepsilon)$.

A comparator using an indirect method of measurement was calibrated for different transformation ratios of reference and DUT transformer. Table 5 shows the deviations of $\varepsilon$ and $\Delta \varphi$ from their reference values as a function of the voltages in the channels $N$ and $X$. Variations of $-1 \% \leq \varepsilon \leq 1 \%$ and $-100^{\prime} \leq \Delta \varphi \leq 100^{\prime}$ were shown not to have any effect on their deviations from their reference values, as expected for this comparator. 
Table 3: Expected properties of comparators using an indirect method of measurement and corresponding checks.

\begin{tabular}{|c|c|}
\hline Property & $\begin{array}{l}\text { Phase error of one channel independent of phase and amplitude of the other channel } \\
\text { Phase error function of range but constant within range }\end{array}$ \\
\hline Check & $\begin{array}{l}\text { Vary phase of signal in other channel while keeping phase in first channel constant } \\
\text { Vary amplitude }\end{array}$ \\
\hline Example & $\begin{array}{l}\text { Vary phase of } X \text { signal while keeping phase of } N \text { signal constant } \\
\text { Repeat for different amplitudes of } N \text { and } X\end{array}$ \\
\hline Type test & Valid for the lifetime of the comparator \\
\hline Property & Actual value phase offset \\
\hline Check & $\begin{array}{l}\text { Apply signal to channel, measure phase error } \\
\text { Repeat for all ranges }\end{array}$ \\
\hline Example & $\begin{array}{l}\text { Set one amplitude of } N \text { signal for each range while keeping } X \text { signal constant } \\
\text { Set one amplitude of } X \text { signal for each range while keeping } N \text { signal constant }\end{array}$ \\
\hline Example & $\begin{array}{l}\text { Set one amplitude of } N \text { signal for each range while synchronising to PPS signal } \\
\text { Set one amplitude of } X \text { signal for each range while synchronising to PPS signal }\end{array}$ \\
\hline $\begin{array}{r}\text { Example } \\
\text { Calibration }\end{array}$ & $\begin{array}{l}\text { Set one amplitude of } N \text { and } X \text { signals at the same time for each range while synchronising to PPS signal } \\
\text { To be repeated at regular intervals }\end{array}$ \\
\hline Property & $\begin{array}{l}\text { Amplitude error independent of amplitude of the other channel } \\
\text { Amplitude measurement linear within ranges }\end{array}$ \\
\hline Check & $\begin{array}{l}\text { Vary amplitude in small steps within range, measure amplitude } \\
\text { Repeat for all ranges } \\
\text { Repeat for all channels }\end{array}$ \\
\hline Example & $\begin{array}{l}\text { Vary amplitude of } N \text { signal while keeping } X \text { signal constant } \\
\text { Vary amplitude of } X \text { signal while keeping } N \text { signal constant }\end{array}$ \\
\hline Type test & Valid for the lifetime of the comparator \\
\hline Property & Actual value of amplitude (offset and gain error) \\
\hline Check & $\begin{array}{l}\text { Apply minimum, average and maximum amplitude within range, measure amplitude } \\
\text { Repeat for all ranges }\end{array}$ \\
\hline Example & Vary amplitude of $N$ and $X$ signals at the same time \\
\hline Calibration & To be repeated at regular intervals \\
\hline Property & Calculation of ratio error exact within numeric resolution \\
\hline Check & $\begin{array}{l}\text { During all previous tests, readout amplitudes of } N \text { and } X \text { as well as ratio error from comparator } \\
\text { Calculate ratio error based on amplitudes of } N \text { and } X \\
\text { Compare calculated ratio error with ratio error readout from the comparator }\end{array}$ \\
\hline Type test & Valid for the lifetime of the comparator's firmware \\
\hline Property & Calculation of phase displacement exact within numeric resolution \\
\hline Check & $\begin{array}{l}\text { During all previous tests, readout phases of } N \text { and } X \text { as well as phase displacement from comparator } \\
\text { Calculate phase displacement based on phases of } N \text { and } X \\
\text { Compare calculated phase displacement with phase displacement readout from the comparator }\end{array}$ \\
\hline Type test & Valid for the lifetime of the comparator's firmware \\
\hline Property & Calculation of phase error exact within numeric resolution \\
\hline Check & $\begin{array}{l}\text { Set rated phase offset and rated delay time [9] to random values } \\
\text { During all previous tests, readout phases displacement as well as phase error from comparator } \\
\text { Calculate phase error based on phase displacement } \\
\text { Compare calculated phase error with phase error readout from the comparator }\end{array}$ \\
\hline Type test & Valid for the lifetime of the comparator's firmware \\
\hline
\end{tabular}

A bridge was calibrated the same way. The deviations of $\varepsilon$ and $\Delta \varphi$ from their reference values depend on both $\varepsilon$ and $\Delta \varphi$, as discussed above. The calibration at $\varepsilon=\Delta \varphi=0$ shows that a well-designed adaptation transformer yields better results than the analogue frontend and the analogue-to-digital converter of the comparator using an indirect method of measurement. Contrary to the comparator using an indirect method of measure- ment, the bridge shows a clear effect when varying $-1 \% \leq$ $\varepsilon \leq 1 \%$ and $-100^{\prime} \leq \Delta \varphi \leq 100^{\prime}$. However, the adaptation transformer is only subject to one signal and cannot be influenced by the other signal. Tables 6 and 7 show the differences in the dependency on $-1 \% \leq \varepsilon \leq 1 \%$ and $-100^{\prime} \leq \Delta \varphi \leq 100^{\prime}$ due to the adaptation transformer. They are smaller than the measurement uncertainty. 
Table 4: Calibration of different comparator models. The rows labelled "max" show the values for the maximum of $\varepsilon$ and $\Delta \varphi$, respectively, in the calibration programme, the rows labelled "min" those for the minimum. The rows labelled " 0 " show the values for $\varepsilon=\Delta \varphi=0$. Models $\mathrm{A}$ and $\mathrm{D}$ are bridges. The other models use an indirect method of measurement.

\begin{tabular}{|c|c|c|c|c|c|}
\hline \multirow[t]{3}{*}{ Model } & & \multicolumn{4}{|c|}{ Deviation with respect to reference value for } \\
\hline & & \multirow{2}{*}{$\frac{\varepsilon(\varepsilon)}{10^{-6}}$} & \multirow{2}{*}{$\frac{\Delta \varphi(\varepsilon)}{\prime}$} & \multirow{2}{*}{$\frac{\varepsilon(\Delta \varphi)}{10^{-6}}$} & \multirow{2}{*}{$\frac{\Delta \varphi(\Delta \varphi)}{\prime}$} \\
\hline & & & & & \\
\hline \multirow[t]{3}{*}{ A } & $\max$ & 2 & 0.09 & -45 & 0.31 \\
\hline & 0 & 2 & 0.00 & 2 & 0.00 \\
\hline & $\min$ & -97 & -0.14 & 14 & -0.29 \\
\hline \multirow[t]{3}{*}{ B } & $\max$ & -5 & 0.01 & -2 & 0.02 \\
\hline & 0 & -2 & 0.02 & -2 & 0.02 \\
\hline & $\min$ & 0 & 0.02 & -2 & 0.01 \\
\hline \multirow[t]{3}{*}{$\mathrm{C}$} & $\max$ & -2 & 0.01 & -2 & 0.02 \\
\hline & 0 & -1 & 0.02 & -1 & 0.02 \\
\hline & $\min$ & -1 & 0.01 & -2 & 0.01 \\
\hline \multirow[t]{3}{*}{ D } & $\max$ & 18 & 0.10 & -45 & 0.11 \\
\hline & 0 & 4 & 0.00 & 4 & 0.00 \\
\hline & $\min$ & -2 & -0.12 & 10 & -0.20 \\
\hline \multirow[t]{3}{*}{$\mathrm{E}$} & $\max$ & 0 & 0.05 & -4 & 0.05 \\
\hline & 0 & -1 & -0.04 & 0 & -0.04 \\
\hline & $\min$ & -4 & -0.04 & -4 & -0.04 \\
\hline \multirow[t]{3}{*}{$F$} & $\max$ & -1 & 0.01 & 2 & 0.01 \\
\hline & 0 & 0 & 0.00 & 0 & 0.00 \\
\hline & $\min$ & 0 & 0.01 & 1 & 0.02 \\
\hline \multirow[t]{3}{*}{ G } & $\max$ & 0 & 0.15 & 0 & 0.15 \\
\hline & 0 & 0 & 0.14 & 1 & 0.14 \\
\hline & $\min$ & -5 & 0.14 & 0 & 0.14 \\
\hline \multicolumn{2}{|c|}{ Uncertainty } & 26 & 0.09 & 26 & 0.09 \\
\hline
\end{tabular}

Table 5: Calibration of a comparator using an indirect method of measurement: Linearity within a range.

\begin{tabular}{|c|c|c|c|}
\hline \multicolumn{2}{|c|}{ Settings } & \multicolumn{2}{|c|}{$\begin{array}{l}\text { Deviation } \\
\text { with respect to reference value for }\end{array}$} \\
\hline$U_{\mathrm{N}}$ & $\overline{U_{x}}$ & $\bar{\varepsilon}$ & $\Delta \varphi$ \\
\hline $\mathbf{v}$ & $\mathbf{v}$ & $10^{-6}$ & 1 \\
\hline 125 & 100 & 10 & 0.00 \\
\hline 100 & 100 & -1 & 0.02 \\
\hline 50 & 100 & -16 & -0.02 \\
\hline \multicolumn{2}{|c|}{ Uncertainty } & 26 & 0.09 \\
\hline
\end{tabular}

Table 6: Calibration of a bridge: Effect of the adaptation transformer as a function of the ratio error $\varepsilon$ with $\Delta \varphi=0$.

\begin{tabular}{|c|c|c|c|c|}
\hline \multirow{2}{*}{$\frac{\text { Setting }}{\varepsilon / \%}$} & \multicolumn{2}{|c|}{$\begin{array}{l}\text { Variation of } \varepsilon / 10^{-6} \\
\text { with respect to } U_{\mathrm{N}}\end{array}$} & \multicolumn{2}{|c|}{$\begin{array}{l}\text { Variation of } \Delta \varphi /^{\prime} \\
\text { with respect to } U_{\mathrm{N}}\end{array}$} \\
\hline & $\overline{50 \mathrm{~V}}$ & $125 \mathrm{~V}$ & $50 \mathrm{~V}$ & $\overline{125 V}$ \\
\hline 1.00 & 3 & -2 & -0.12 & 0.00 \\
\hline 0.10 & 5 & -2 & -0.13 & 0.02 \\
\hline 0.01 & 6 & -1 & -0.13 & 0.02 \\
\hline 0.00 & 3 & -7 & -0.12 & 0.02 \\
\hline-0.01 & 4 & -3 & -0.13 & 0.01 \\
\hline-0.10 & 6 & -3 & -0.13 & 0.02 \\
\hline-1.00 & 15 & 4 & -0.11 & 0.02 \\
\hline
\end{tabular}

Table 7: Calibration of a bridge: Effect of the adaptation transformer as a function of the phase displacement $\Delta \varphi$ with $\varepsilon=0$.

\begin{tabular}{|c|c|c|c|c|}
\hline \multirow{2}{*}{$\frac{\text { Setting }}{\Delta \varphi /^{\prime}}$} & \multicolumn{2}{|c|}{$\begin{array}{l}\text { Variation of } \varepsilon / 10^{-6} \\
\text { with respect to } U_{N}\end{array}$} & \multicolumn{2}{|c|}{$\begin{array}{l}\text { Variation of } \Delta \varphi /^{\prime} \\
\text { with respect to } U_{\mathrm{N}}\end{array}$} \\
\hline & $\overline{50 \mathrm{~V}}$ & $\overline{125 V}$ & $50 \mathrm{~V}$ & $125 \mathrm{~V}$ \\
\hline 100 & 5 & 2 & -0.20 & 0.00 \\
\hline 10 & 4 & -3 & -0.13 & 0.01 \\
\hline 1 & 2 & -4 & -0.15 & 0.00 \\
\hline 0 & 3 & -7 & -0.12 & 0.02 \\
\hline-1 & 2 & -4 & -0.13 & 0.01 \\
\hline-10 & 5 & -4 & -0.12 & 0.02 \\
\hline-100 & 2 & -9 & -0.10 & 0.10 \\
\hline
\end{tabular}

\section{Conclusion}

It is always important to take the operation principle into account when defining calibration programmes. The example discussed here is comparators for instrument transformers. Some are bridges, but most new commercial comparators use an indirect method of measurement. An adapted calibration programme gives traceability to the full operating range and not just to the part that is in common with traditional bridges. Since many properties are not subject to ageing, this article proposed defining a type test programme - to be carried out once - and a calibration programme - to be repeated regularly. The programmes were derived from purely theoretical knowledge about the instrument. Experiments with real instruments confirmed the assumptions.

Acknowledgment: This research and the related metrology services are part of the European Metrology Network for Smart Electricity Grids [21], the single point of contact across Europe that provides stakeholder support for measurement challenges in the realisation of smart electricity grids.

Funding: This research was developed in the framework of the 16ENG04 MyRails project [22]. The latter received funding from the EMPIR programme co-financed by the Participating States and the European Union's Horizon 2020 research and innovation programme.

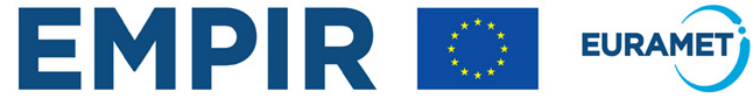

The EMPIR initiative is co-funded by the European Union's Horizon 2020 research and innovation programme and the EMPIR Participating States 


\section{References}

1. IEC 60050: International Electrotechnical Vocabulary, 2020-11-09.

2. S. Siegenthaler and C. Mester. A computer-controlled calibrator for instrument transformer test sets. IEEE Transactions on Instrumentation and Measurement, vol. 66 no. 6, June 2017, pp. 1184-1190.

3. IEC 61869-1:2007: Instrument transformers - Part 1: General requirements.

4. IEC 61850-9-2:2011: Communication networks and systems for power utility automation - Part 9-2: Specific communication service mapping (SCSM) - Sampled values over ISO/IEC 8802-3.

5. K. Draxler et al. Results of an International Comparison of Instrument Current Transformers up to $10 \mathrm{kA}$ and $50 \mathrm{~Hz}$ Frequency. In: Conference on Precision Electromagnetic Measurements CPEM, July 2018.

6. IEC/IEEE TS CD 61869-105:2018 (38/567/CD): Instrument transformers - Part 105: Uncertainty evaluation in the calibration of instrument transformers.

7. A. P. Chattock. On a magnetic potentiometer. Philosophical Magazine and Journal of Science, vol. XXIV, no. 5, 1887, pp. 94-96.

8. C. Mester et al. Einführung in die rückführbare Messung von Power Quality. tm - Technisches Messen, Band 85, Heft 12, 2018, pp. 738-745.

9. IEC 61869-6:2016: Instrument transformers - Part 6: Additional general requirements for low-power instrument transformers.

10. IEC CD 61869-1:2020 (38/631/CD): Instrument transformers -Part 1: General requirements.

11. L. Hentgen and C. Mester. Sampling AC signals: Comparison of fitting algorithms and FFT. In: Conference on Precision Electromagnetic Measurements CPEM, July 2018.

12. IEC 61869-9:2016: Instrument transformers - Part 9: Digital interface for instrument transformers.

13. IEC FDIS 61869-13:2020 (38/634/FDIS): Instrument Transformers -- Part 13: Standalone Merging Unit.

14. C. Mester. Timestamping type 3458A multimeter samples. In: Conference on Precision Electromagnetic Measurements CPEM, July 2018.

15. J. Feng et al. Time Latency of DC Input Path for 3458A Sampling Multimeter. In: Conference on Precision Electromagnetic Measurements CPEM, July 2018.

16. G. Crotti et al. A method for the measurement of digitizers' absolute phase error. Journal of Physics: Conference Series, vol. 1065, 2018.

17. G. Crotti et al. Measurement of the absolute phase error of digitizers. IEEE Transactions on Instrumentation and Measurement, vol. 68, no. 6, June 2019, pp.1724-1731.

18. J.-P. Braun. Measure of the Absolute Phase Angle of a Power Frequency Sinewave with Respect to UTC. In: Conference on Precision Electromagnetic Measurements CPEM, July 2018.

19. EN 50470: Electricity metering equipment (a. c.). General requirements, tests and test conditions. Metering equipment (class indexes $A, B$ and $C$ ).

20. Verordnung des EJPD vom 26. August 2015 über Messmittel für elektrische Energie und Leistung (EMmV, SR 941.251).
21. European Metrology Network for Smart Electricity Grids. www.euramet.org/european-metrology-networks/smartelectricity-grids/, 2020-11-13.

22. D. Giordano et al. Accurate Measurement of Energy, Efficiency and Power Quality in the Electric Railway System. In: Conference on Precision Electromagnetic Measurements CPEM, July 2018.

\section{Bionotes}

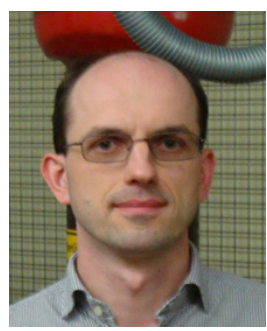

Christian Mester

Federal Institute of Metrology METAS, Lindenweg 50, 3003 Bern, Switzerland christian.mester@metas.ch

Christian Mester was awarded a PhD for his work at CERN, the European Organization for Nuclear Research, in Geneva, Switzerland, by the University of Bonn, Germany, in 2009. After a postdoc at École Polytechnique Fédérale de Lausanne EPFL, in Lausanne, Switzerland, he joined the Electrical Power and Energy laboratory at the Federal Institute of Metrology METAS in Berne, Switzerland, in 2011. His current research activities include the calibration of conventional and non-conventional instrument transformers, power standards and power quality instruments, including applications in charging stations for electric vehicles, as well as sampling procedures and algorithms. 